\title{
Spraying the Replication Probability With Geographic Assistance for Delay Tolerant Networks
}

\author{
Yue Cao, Zhili Sun, Ning Wang \\ Centre for Communication Systems Research \\ University of Surrey, Guildford, UK \\ \{Y.Cao; Z.Sun; N.Wang\}@ surrey.ac.uk
}

\begin{abstract}
Receiving great interest from the research community, Delay Tolerant Networks (DTNs) are a type of Next Generation Networks (NGNs) proposed to bridge communication in challenged environments. In this paper, the message replication probability is proportionally sprayed for efficient routing mainly under sparse scenario. This methodology is different from the spray based algorithms using message copy ticket to control replication. Our heuristic algorithm aims to overcome the scalability of spray based algorithms since to determine the initial value of copy ticket requires the assumption that either the number of nodes known in advance or the underlying mobility model follows the Random WayPoint (RWP) characteristic.

Specifically, in combining with the assistance of geographic information to estimate the movement range of destination, our routing decision is based on the encounter angle between pairwise nodes, and dynamically switched between our designed two routing phases named as geographic replication and replication probability spray. Furthermore, messages are under transmission with the consideration of redundancy pruning. Simulation results show our heuristic algorithm outperforms other well known algorithms in terms of delivery ratio, transmission overhead, average latency as well as buffer occupancy time.
\end{abstract}

\section{INTRODUCTION}

A set of improved versions of Spray-and-Wait [1] have been proposed for efficient routing in Delay Tolerant Networks (DTNs) [2] during the last few years. The core concept is to define an initial copy ticket value for each generated message to limit the number of replications, and enable intermediate nodes carrying these message copy tickets to encounter destination for efficient routing.

However, the primary problem for these algorithms is how to determine the initial value of copy ticket if the underlying mobility does not follow the Random WayPoint (RWP), or the number of nodes is unknown in reality. In this paper, we adopt the mechanism to spray the message replication probability rather than copy ticket, to overcome the limitation of spray based algorithm since to determine the value of copy ticket is not taken into account by our proposed algorithm. This approach is more feasible in reality to achieve the similar low transmission overhead of spray based algorithms particularly under sparse scenario.

Considering moving direction, we further propose it is unnecessary to replicate message to the intermediate node moving away from destination. To replicate message towards its destination, message is processed with geographic consideration if the encounter angle between pairwise nodes is large since they are moving with different direction. However, the opportunistic scenario of DTNs is assumed to be sparse or highly mobile, which is unrealistic to obtain the realtime location of destination because of the long delay for information request or rapid change of location. In light of this, we propose to estimate the movement range of destination and promote message replication based on the proximity to this range. In addition, pairwise nodes are moving with similar direction if their encounter angle is small, thus the message replication is probabilistic for redundancy reduction in this case, where this replication probability is proportionally distributed according to our proposed utility metric.

The contributions in this paper are as follows:

- By means of our defined utility metric, a methodology to spray the message replication probability rather than message copy ticket is proposed, thus the number of replications is unlimited.

- With the assistance of geographic information, a heuristic routing algorithm consisting of two routing phases is proposed. Based on the scheduling priority for these two routing phases, messages are under prioritized transmission to tolerate frequent disruption due to high mobility with the consideration of redundancy reduction.

- We simulate Epidemic [3], Adaptive Epidemic [4], POR [5] (POR is based on realtime location information) and EBR [6] (EBR is a powerful spray based algorithm) for performance comparison under the Helsinki city scenario.

We firstly argue to spray the message replication probability is a potential approach to achieve efficiency if the existing spray based algorithms are limited by their requirement. Besides, it is also difficult to obtain the realtime location in DTNs due to sparse network density and high mobility.

\section{RELATED WORK}

In this paper, we omit the routing algorithms with the assistance infrastructure such as message ferry/data mule and throwbox/gateway, since they either focus on route design or location deployment for infrastructure.

Without such assistance, the early stage algorithms are based on the utility metric to select candidate node, using single copy based forwarding [7].

Inherently, it is effective to replicate message regardless of candidate node selection for achieving maximum delivery ratio under even with redundancy [3]. Motivated by this limitation, 
Spray-and-Wait [1] limits the number of replications by a copy ticket defined for each generated message.

Up to now, there have been some evolutions of Spray-andWait. In [8], message is sprayed to the encountered node qualified by utility metric. The work in [9] focuses on how to dynamically determine the value of copy ticket. Based on oracle information about network, copy ticket is proportionally sprayed in [6]. In order to achieve the target latency, to control the spray depth is proposed in [10].

Inherently, these spray algorithms focus on how to utilize the limited number of replications. Furthermore, the work in [11] has proved the optimal approach to proportionally spray the copy ticket relies on the initialized value of copy ticket. However, to determine this value is always a challenging problem in reality rather than RWP scenario, since the initialization is only based on the assumption of exponentially distributed inter meeting time. As well, it is also limited by the lack of knowledge about the number of nodes in the network, as mentioned in [1].

Another methodology for redundancy reduction is to replicate message based on utility metric. In [12], message is replicated using encounter probability with aging and transitivity consideration. Based on the distance towards destination, geographic algorithm [5] assumes the realtime location is shared by all of the nodes in the networks or the delay to obtain such information can be ignored.

\section{Our Proposed Algorithm}

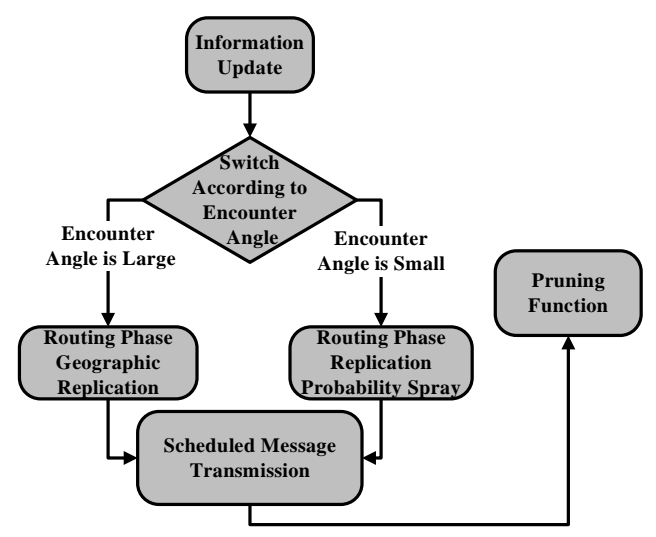

Fig. 1. Function Flowchart

The overall function flowchart is illustrated in Fig.1 and the detail of the design is introduced in the following subsections. Briefly, driven by the encounter angle between pairwise encountered node, the key insight of our heuristic algorithm is to probabilistically replicate message according to the proportionally distributed probability (we name as replication probability spray) if pairwise nodes encounter with a small angle. Otherwise, to replicate message towards its destination in case of large encounter angle is for sufficient replication given the limited message lifetime, named as geographic replication. The designed scheduled message transmission and the specific process after transfer further promote the performance with the consideration of load balancing and redundancy pruning.

\section{A. Definition of Utility Metric}

Considering mobility factor, we address three conventional metrics of pairwise encountered nodes $N_{i}$ and $N_{j}$, which are history encounter count $C_{i, j}$, history encounter duration $D_{i, j}$ and history inter meeting time $I_{i, j}$, where $i, j \in S$ and $S$ is the total number of nodes in the networks.

Particularly, the history inter meeting time consists of history encounter duration $D_{i, j}$ and disruption time until pairwise nodes encounter again. As illustrated in Fig.2, a high ratio of $\frac{D_{i, j}}{I_{i, j}}$ indicates a more efficient inter meeting time since more messages can be transmitted during this encounter duration. We propose an accumulative formula to smooth the factor of large variation within the number of encounters, where the utility $U_{i, j}$ is defined as:

$$
U_{i, j}=\frac{\sum_{k=2}^{C_{i, j}}\left(\frac{D_{i, j}^{k-1}}{I_{i, j}^{k}}\right)}{\left(C_{i, j}-1\right)}
$$

where $D_{i, j}^{k-1}$ is the encounter duration before the $k^{t h}$ encounter and it is valid after the first encounter. With the time elapsing this property is useful since the node experiences a large number of encounters is more likely to successfully route the message to the final destination than those who have infrequent encounters.

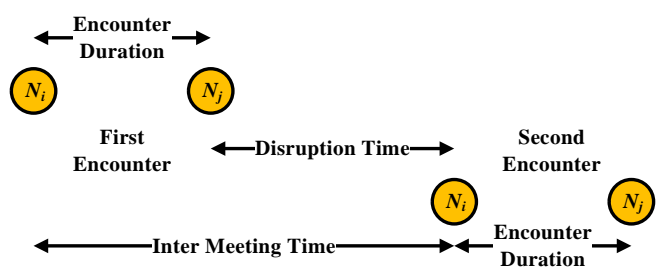

Fig. 2. Encounter History of Pairwise Nodes

Inherently, to estimate the delivery potential of encountered nodes based on local knowledge is unreasonable since the factor of other history encountered nodes is ignored. To overcome this limitation, pairwise nodes would calculate and update their $U_{i, j}$ for each other. Afterwards, they would add their local routing information to each other for the purpose of extending knowledge. To this end, they can obtain the knowledge from their neighbors' history encounter information.

Based on the above analysis, an improved utility $U_{i, j}^{\prime}$ is proposed:

$$
U_{c, d}^{\prime}=\frac{\sum_{k=1}^{n} U_{c(k), d}+U_{c, d}}{\xi+1}
$$

where $n$ is the number of history encountered nodes $c(n)$ of current carrier $c, d$ is the corresponding destined node. In detail, $\xi$ is initialized with zero and increased by one if $c(n)$ contains $U_{c(n), d}$ for $d$. Therefore, the local node would obtain an abstract average knowledge for $d$ not only based on itself but also based on the history information from its neighbors. As an example in TABLE I, the $U_{6,2}^{\prime}$ for $N_{2}$ based on the view of $N_{6}$ is calculated as:

$$
U_{6,2}^{\prime}=\frac{U_{1,2}+U_{5,2}+U_{6,2}}{2+1}=\frac{0.6+0.3+0}{2+1}=0.3
$$


TABLE I

EXAmple of Routing TABLE ReCORDEd By $N_{6}$

\begin{tabular}{|l|c|c|}
\hline \multirow{3}{*}{ current carrier $N_{6}$} & $U_{6,1}$ & 0.4 \\
& $U_{6,5}$ & 0.2 \\
& $U_{6,2}$ & 0.0 \\
\hline \multirow{3}{*}{ history encountered $N_{1}$ of $N_{6}$} & $U_{1,6}$ & 0.4 \\
& $U_{1,2}$ & 0.6 \\
& $U_{1,4}$ & 0.3 \\
\hline & $U_{5,2}$ & 0.3 \\
history encountered $N_{5}$ of $N_{6}$ & $U_{5,8}$ & 0.1 \\
& $U_{5,6}$ & 0.2 \\
\hline
\end{tabular}

\section{B. Information Update}

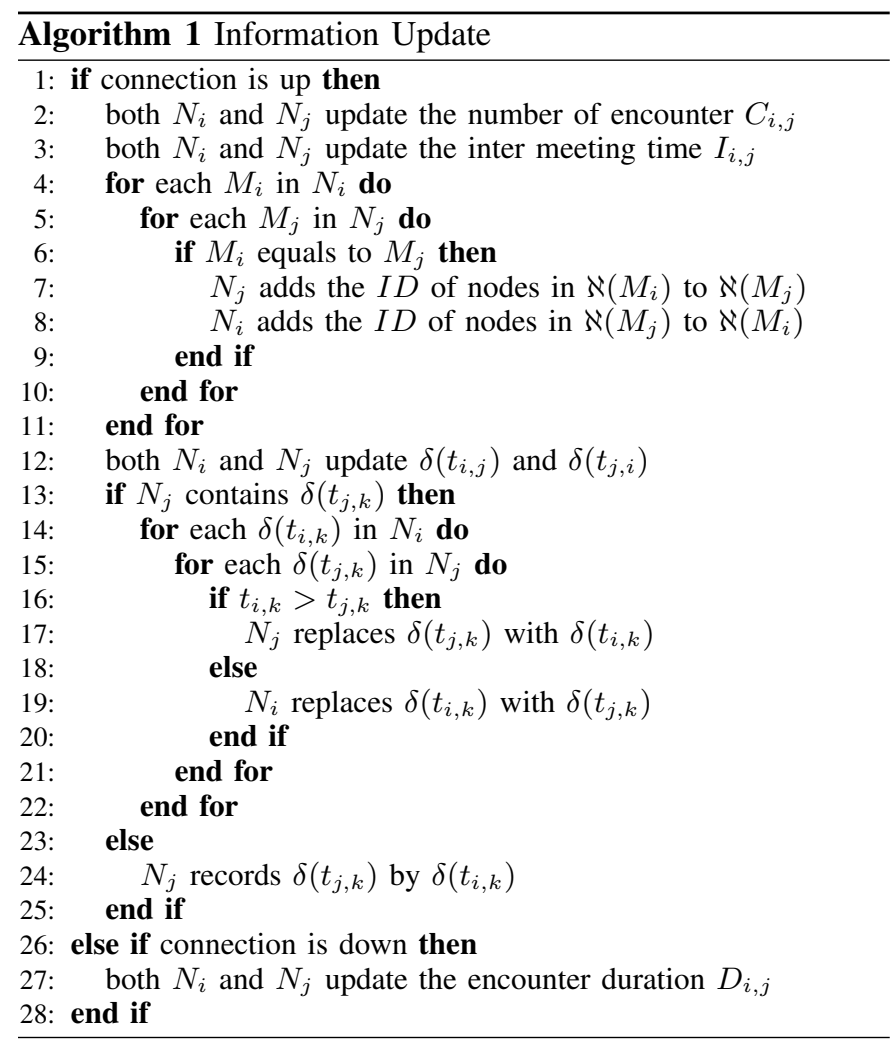

TABLE II

INFORMATION FORMAT OF $\delta\left(t_{i, k}\right)$

\begin{tabular}{|l|l|l|l|}
\hline Node ID & History Location & History Speed & Encounter Time \\
\hline
\end{tabular}

Borrowing the ID identified for each node, the carrier count $\aleph(M)$ is denoted as the number of nodes have carried message $M$. In addition, we assume each mobile node can only obtain its local realtime location by means of the Global Positioning System (GPS) without consideration of GPS error. Specifically, as illustrated in TABLE II, the definition of geographic information $\delta\left(t_{i, k}\right)$ is about the history location $L\left(t_{i, k}\right)$ of $N_{k}$ together with its speed $S_{k}$ obtained by $N_{i}$ at their encounter time $t_{i, k}$. This information is updated to the value obtained at more recent time if both of pairwise nodes encountered $N_{k}$. Otherwise one of them records other's information for backup. The current $\delta\left(t_{i, j}\right)$ and $\delta\left(t_{j, i}\right)$ of pairwise encountered nodes $N_{i}$ and $N_{j}$ are also exchanged for information update. A detail process regarding information update is illustrated in Algorithm 1.

In particular, the information update activated when encounter event happens is essential for all of the routing algorithms in DTNs, rather than the periodic update for traditional algorithms in Mobile Ad hoc NETworks (MANETs).

\section{Routing Framework}

With an appropriate time window $\triangle T$ for the location update, we adopt the mobility vector to indicate the trajectory. Specifically, $\triangle V_{i}$ is the variation of $N_{i}$ 's mobility vector within previous $\triangle T$, which consists of the specific variation of location $\triangle X_{i}$ and $\triangle Y_{i}$. Therefore, the encounter angle $\theta$ between $N_{i}$ and $N_{j}$ can be calculated by geometry.

In our routing framework, messages are processed respectively by replication probability spray and geographic replication phase according to the decision made from the encounter angle $\theta \in[0, \pi]$ between pairwise nodes:

$$
\theta= \begin{cases}\left|\nu_{i}-\nu_{j}\right| & \text { if }\left|\nu_{i}-\nu_{j}\right| \leq \pi \\ 2 \pi-\left|\nu_{i}-\nu_{j}\right| & \text { else }\end{cases}
$$

Where $\nu_{i}$ and $\nu_{j}$ are the moving direction of $N_{i}$ and $N_{j}$. Generally, if $\theta$ is large enough, it makes sense to adopt geographic replication since it only replicates message towards destination for sufficient delivery. Relatively, it is unnecessary to replicate message by geographic replication if $\theta$ is quite small since pairwise nodes are moving with similar direction. In light of this, we adopt the replication probability spray for efficiency. In particular, a large value of $\frac{\theta}{\pi}$ indicates a large encounter angle and vice versa.

1) Geographic Replication: Suppose the history location of destination $N_{d}$ obtained at more recent time is $L(d)_{i, j}$ since $\delta\left(t_{i, d}\right)$ and $\delta\left(t_{j, d}\right)$ for $N_{d}$ are consistent after update. Then the estimated movement range of $N_{d}$ is defined as a virtual circle of which the radius is $\Re$, under the assumption of constant moving speed $S_{d}$ :

$$
\Re=\left(t_{\text {cur }}-t_{d}\right) * S_{d}
$$

where $t_{c u r}$ is the current time in the network and $t_{d}$ is the more recent time value between $t_{i, d}$ and $t_{j, d}$. The distance from the location of $N_{j}$ to $L(d)_{i, j}$ is denoted as $d i s t_{j \rightarrow d}$ at $t_{\text {cur }}$.

The condition dist $_{j \rightarrow d}<\Re$ indicates $N_{j}$ is within the movement range estimated for $N_{d}$, thus message $M$ carried by $N_{i}$ is replicated for sufficient delivery. As $N_{j}$ is out of such range given $d i s t_{j \rightarrow d} \geq \Re, M$ is replicated to $N_{j}$ only if the angle $\phi$ between its current moving direction $\nu_{j} \in[0,2 \pi]$ and the line connection of $d i s t_{j \rightarrow d}$ is smaller than $\frac{\pi}{2}$. As an example illustrated in Fig.3, the current moving direction of $N_{j}$ is parallel to the tangent of virtual circle estimated for $N_{d}$, thus it makes sense that $N_{j}$ will not encounter $N_{d}$, since the encounter only happens if the current moving direction of $N_{j}$ follows the secant of this circle. 


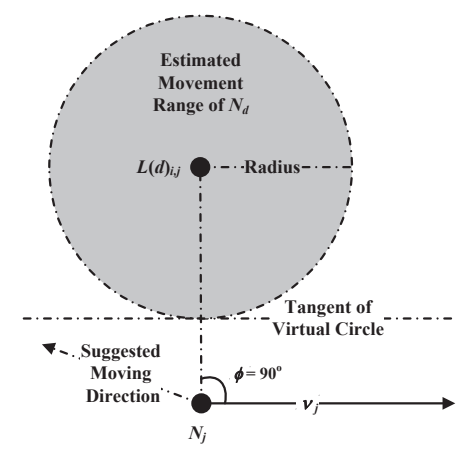

Fig. 3. An Example of Geographic Replication

2) Replication Probability Spray: The novelty of replication probability spray is to proportionally spray the replication probability of $M$ according to our defined utility metric for its destination $N_{d}$. Generally, the initial replication probability for each generated message is set to 1 . If pairwise nodes $N_{i}$ and $N_{j}$ encounter, the replication probability $R P\left(M_{i}\right)$ of $M$ carried by $N_{i}$ and the $R P\left(M_{j}\right)$ of the replicated $M$ to $N_{j}$ are calculated according to a weighted distribution approach:

$$
\begin{aligned}
& R P\left(M_{i}\right)=U_{i, d}^{\prime} *\left[\frac{R P\left(M_{i}\right)}{U_{i, d}^{\prime}+U_{j, d}^{\prime}}\right] \\
& R P\left(M_{j}\right)=U_{j, d}^{\prime} *\left[\frac{R P\left(M_{i}\right)}{U_{i, d}^{\prime}+U_{j, d}^{\prime}}\right]
\end{aligned}
$$

This formula guarantees a large replication probability of $M$ is sprayed to the candidate node with higher utility metric for $N_{d}$. In addition, if both $N_{i}$ and $N_{j}$ already carry $M$, the replication probability is firstly normalized as the maximum value between $R P\left(M_{i}\right)$ and $R P\left(M_{j}\right)$ to guarantee a sufficient probability for subsequent replication, these values are recalculated according to equation (6) and equation (7).

3) Scheduled Message Transmission: As there are two routing phases designed, to determine their scheduling priority is essential. In light of this, we design a dynamic scheduling mechanism with the scheduling priority $S P$ defined as:

$$
S P=\sum_{k=1}^{m} G_{(M, j)}
$$

where $m$ is the number of enqueued messages processed by each routing phase. We assume the determined candidate node $N_{j}$ has a gravitation for message $M$ carried by $N_{i}$. Thus the gravitation $G_{(M, j)}$ is defined as:

$$
G_{(M, j)}=\frac{\Psi_{M} * \Psi_{j}^{\text {free }}}{\left(T_{M}^{i n i}-T_{M}^{c u r}\right)^{2}}
$$

where $\Psi_{M}$ is the size of $M$ and $\Psi_{j}^{\text {free }}$ is the free buffer space of $N_{j} . T_{M}^{i n i}$ is the initial lifetime of $M$ and $T_{M}^{c u r}$ is its current lifetime. In particular, this formula not only balances the traffic load by taking into account free buffer size, but also it more focuses on achieving less delivery latency since the message lifetime squarely affects its priority. If the distance regarding message lifetime is large, accordingly $N_{j}$ as the next

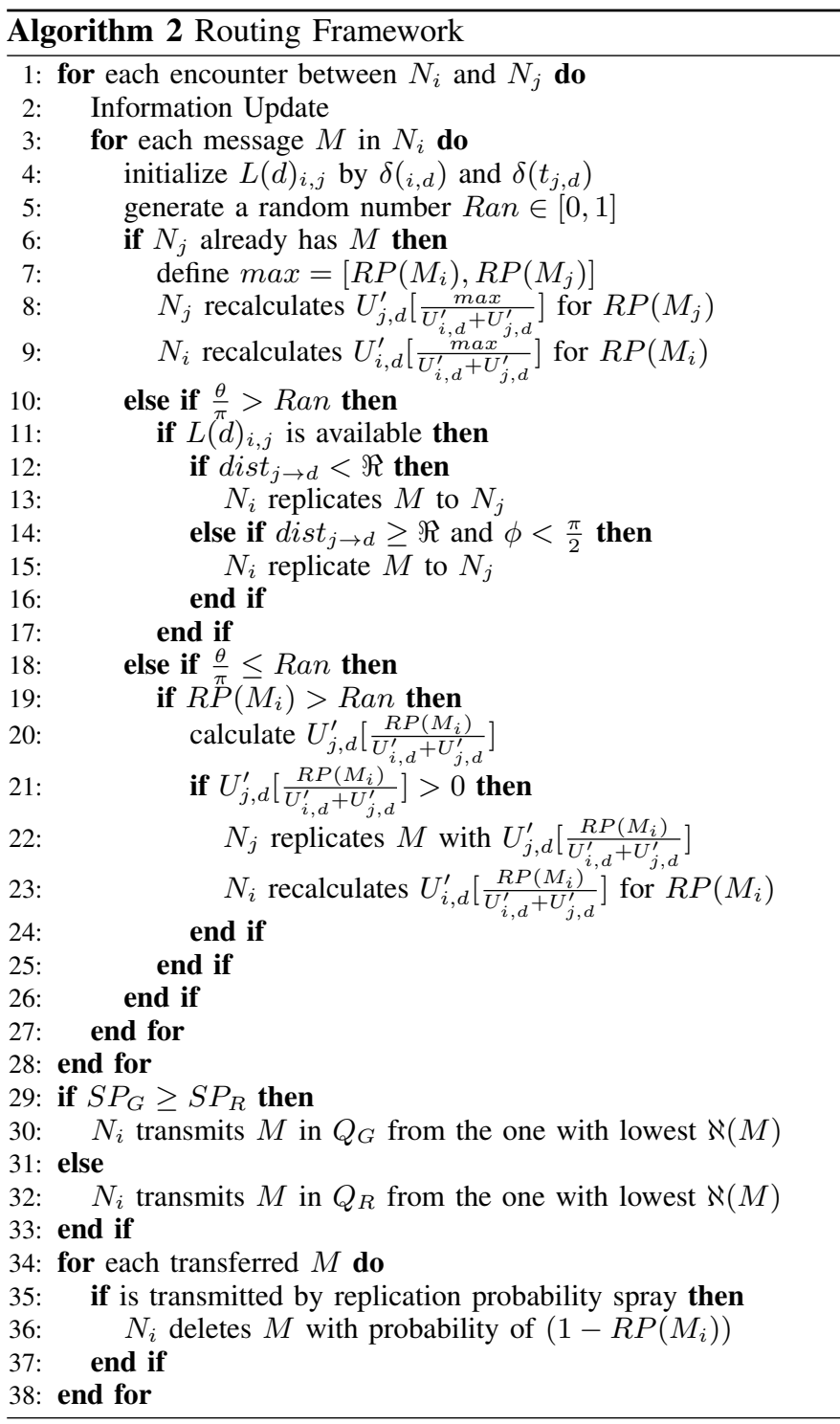

hop might not receive $M$ since $M$ might be expired before message transfer, as such $G_{(M, j)}$ is small.

Therefore the $S P_{G}$ for geographic approach and the $S P_{R}$ for replication probability spray can be calculated respectively. Specifically, the queue $Q_{g e o}$ for geographic replication phase and the $Q_{r p s}$ for replication probability spray phase is scheduled based on a larger value of $S P$, where the calculation of $S P$ is reset after each successful transfer of each message.

With respect to the messages transmitted using same routing phase, we utilize the previously defined carrier count $\aleph(M)$ for their priority, since this updated information in Algorithm 1 is used to estimate the number of nodes have carried $M$. Therefore, message with the least $\aleph(M)$ is regarded as the highest priority for transmission, since it is most fresh in the network.

4) Pruning Function: Since the messages are replicated according to different routing phases, they are processed respectively after successful transfer. Regarding message repli- 
cated by geographic replication, the current carrier always keeps the original message since it moves with a different direction between encountered node, which guarantees the sufficient message diffusion. Relatively, message processed by replication probability spray is likely to be deleted if its $R P\left(M_{i}\right)$ is quite small after spray process, which implies a large replication probability has been sprayed to a better candidate node moving with the similar direction, thus it is unnecessary for the current carrier to keep message.

\section{PERformance EVAluation}

The results are evaluated by the Opportunistic Network Environment (ONE) [13]. The Helsinki city scenario area is $15.3 \mathrm{~km}^{2}$ where 126 mobile nodes are classified as 80 pedestrians with [0.5-1.5] $/ \mathrm{m}, 40$ cars with $[2.7-13.9] \mathrm{m} / \mathrm{s}$ and 6 trams with [7-10]m/s. The buffer size is set to be large enough so that messages are not dropped due to buffer exhaustion. In particular, each node has an interest to visit some places rather than randomly select the next point based on route. The connectivity is $2 \mathrm{Mb} / \mathrm{s}$ with 20 meters transmission range as the Bluetooth technique. An initial 3600s is used to collect the history information required for routing decision and messages are generated between 3600s and 18000s. The $\triangle T$ for local location update is $1 \mathrm{~s}$. Considering the delay tolerant characteristic of DTNs, the message lifetime is set with 2.5 hours.

\section{A. Algorithms for Comparison}

We compare our heuristic algorithm with Epidemic [3] and Adaptive Epidemic [4] for performance evaluation. As well, with the geographic assistance to enable the message diffusion towards destination, geographic algorithm POR [5] is compared. As mentioned, the heuristic is applied if either the underlying mobility model does not strictly follow RWP, or the number of nodes in the network is unknown in advance, these requirements are difficult for the application of spray based algorithms. In light of this, EBR [6] is selected for comparison where the initial value of copy ticket is set with 13 determined by $10 \%$ number of nodes in the network. This is a recommended value advised in [1] if the knowledge of network mobility model is unavailable. In particular, the realtime location is shared by all of the nodes in POR. The parameter configuration of Adaptive Epidemic and EBR are based their default values. We plot the average value for the results evaluation to smooth the random factor.

\section{B. Evaluation Results}

Given the large enough message lifetime, the overhead ratio $^{2}$ achieved by our algorithm is close to EBR in Fig.4(c), thanks to the proportionally distributed message replication probability assisted by the geographic replication. Inherently, the message replication count is without limitation in our algorithm, whereas to some extent, EBR is limited by the initial value of copy ticket. Therefore, our approach achieves

\footnotetext{
${ }^{2}$ The overhead ratio is given by the ratio between the number of successful relayed messages and the total number of messages delivered.
}

TABLE III

INITIAL SIMULATION CONFIGURATION

\begin{tabular}{|c|c|}
\hline Simulation Time & 21600 seconds (6 Hours) \\
\hline Bandwidth & $2 \mathrm{Mb} / \mathrm{s}$ \\
\hline Transmission Range & $20 \mathrm{~m}$ \\
\hline Buffer Size & $1500 \mathrm{MB}$ \\
\hline Number of Nodes & 126 \\
\hline Message Size & $500 \mathrm{kB} \sim 2 \mathrm{MB}$ \\
\hline Message Generation & Every 9s between 3600s and 18000s \\
\hline Message Lifetime & 2.5 Hours \\
\hline Scenario Mobility & Helsinki City Model \\
\hline
\end{tabular}

lower latency in Fig.4(b) than EBR because of more replications. Assisted by the greedy geographic replication, the delivery ratio in Fig.4(a) is enhanced particularly given small message lifetime. Besides, to delete message with the smallest replication probability contributes to the lowest average buffer time in Fig.4(d) ${ }^{3}$.

Inherently, the alleviated bandwidth contention increases message delivery ratio in Fig.5(a) and overhead in Fig.5(c). Even EBR achieves a slightly less overhead than our proposed algorithm, the significant drawback of EBR is the high latency in Fig.5(b) as well as the buffer time in Fig.5(d). These negative aspect is due to the limited number of replications and the lack of the pruning function. In particular, the increased generation rate does not directly affect the buffer time for all of of compared algorithms since the buffer space is set to be large enough.

The results in Fig.6(a) and Fig.6(c) keep an increment growth since more messages can be delivered because of the increased transmission range. EBR achieves the lowest overhead ratio given the large transmission range, since its number of replications is partially limited by the initial copy tickets. However, the overhead ratio achieved by our algorithm is close to EBR when the transmission range is around 20 meters, this implies our efficiency under sparse scenario. Apart from the overhead ratio, the proposed algorithm still performs well particularly if the transmission range is large enough since the history information is updated more frequently. This is beneficial to the movement range estimation for destination and message deletion. In light of this, the improvement in Fig.6(b) and Fig.6(d) become significant.

\section{CONClusion}

A heuristic routing algorithm for DTNs has been proposed in this paper, which sprays the message replication probability rather than copy ticket. The main motivation is to loose the scalability problem of spray based algorithm, that is to determine the initial value for copy ticket. Based on our defined utility metric for replication probability distribution, the heuristic algorithm dynamically switches between the designed geographic replication phase and the replication probability spray phase according to encounter angle between

\footnotetext{
${ }^{3}$ The average buffer time is defined as the average value of the time period for the carried messages in the buffer of each node.
} 


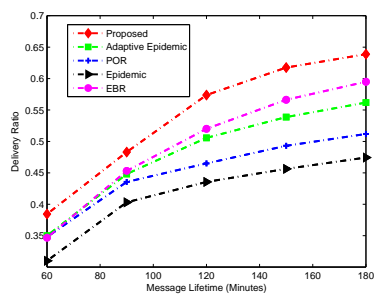

(a) Delivery Ratio

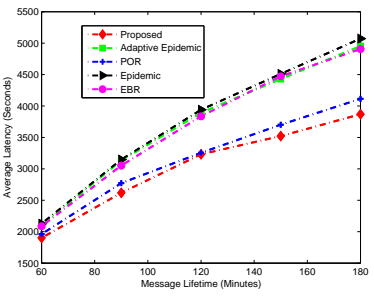

(b) Average Latency

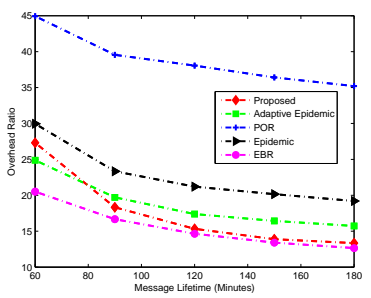

(c) Overhead Ratio

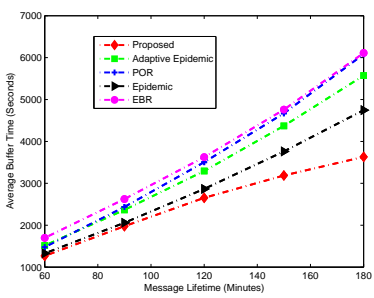

(d) Average Buffer Time

Fig. 4. Effect of Message Lifetime in Case of 20 Meters Transmission Range and 9s Message Generation Rate

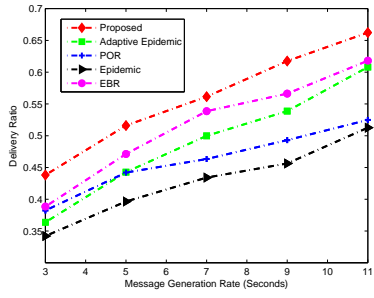

(a) Delivery Ratio

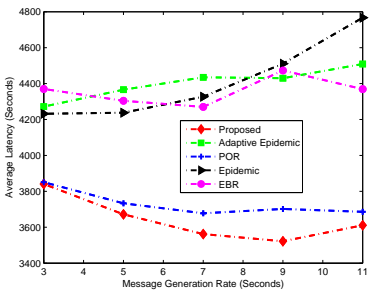

(b) Average Latency

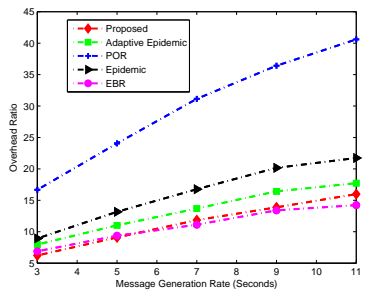

(c) Overhead Ratio

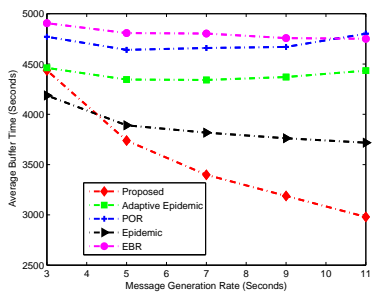

(d) Average Buffer Time

Fig. 5. Effect of Message Generation Rate in Case of 2.5 hours Message Lifetime and 20 Meters Transmission Range

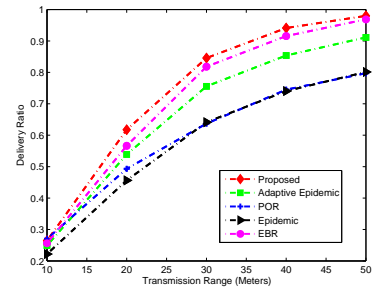

(a) Delivery Ratio

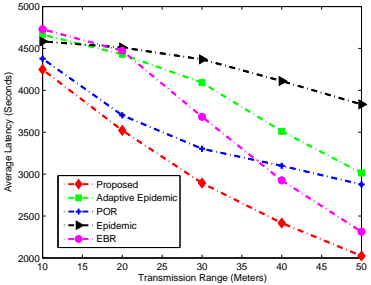

(b) Average Latency

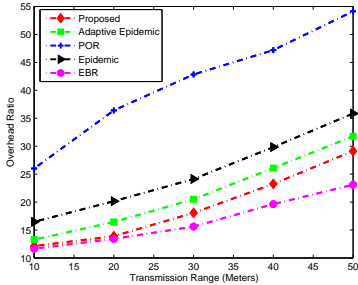

(c) Overhead Ratio

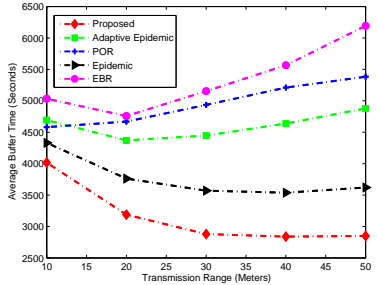

(d) Average Buffer Time

Fig. 6. Effect of Transmission Range in Case of 2.5 Hours Message Lifetime and 9s Message Generation Rate

pairwise nodes. In addition, our algorithm is with prioritized message transmission between each phase as well as the redundancy pruning for performance enhancement. Evaluated under the Helsinki city, the proposed algorithm outperforms the literature algorithms in terms of evaluation metrics as well as low buffer occupancy particularly with small transmission range and relatively sufficient message lifetime, thus is more feasible in sparse DTNs.

Since the idea of the proposed approach is unique and worthwhile investigating given a huge number of existing works, the future work will focus on the deduction of overhead upper bound as well as how to reduce the signalling overhead taking into account the GPS error.

\section{ACKNOWLEDGEMENT}

The funding leading to this work is from the EU FP7 MONET project and EPSRC UK-CHINA Science Bridge UC4G project. The authors wish to acknowledge the support from Datang Wireless Mobile Innovation Center (DWMIC) China Academy of Telecommunication Technology (CATT).

\section{REFERENCES}

[1] T. Spyropoulos, K. Psounis, and C. Raghavendra, "Efficient routing in intermittently connected mobile networks: The multiple-copy case," IEEE/ACM Transactions on Networking, vol. 16, no. 1, pp. $77-90$, 2008.
[2] K. Fall and S. Farrell, "Dtn: an architectural retrospective," IEEE Journal on Selected Areas in Communications, vol. 26, no. 5, pp. 828 -836, 2008.

[3] A. Vahdat and D. Becker, "Epidemic routing for partially-connected ad hoc networks," Duke University Technical Report Cs-2000-06, Tech. Rep., 2000.

[4] F. Hou and X. Shen, "An adaptive forwarding scheme for message delivery over delay tolerant networks," in IEEE GLOBECOM '09, Honolulu, Hawii, USA, 2009.

[5] X. Li, W. Shu, M. Li, H. Huang, and M.-Y. Wu, "Dtn routing in vehicular sensor networks," in IEEE GLOBECOM '08, New Orleans, Louisiana, USA, 2008.

[6] S. Nelson, M. Bakht, and R. Kravets, "Encounter-based routing in dtns," in IEEE INFOCOM '09, Rio de Janeiro, Brazil, 2009.

[7] S. Jain, K. Fall, and R. Patra, "Routing in a delay tolerant network," in ACM SIGCOMM'04, Oregon, Portland, 2004.

[8] T. Spyropoulos, T. Turletti, and K. Obraczka, "Routing in delaytolerant networks comprising heterogeneous node populations," IEEE Transactions on Mobile Computing, vol. 8, no. 8, pp. 1132 -1147, 2009.

[9] E. Bulut, Z. Wang, and B. Szymanski, "Cost-effective multiperiod spraying for routing in delay-tolerant networks," IEEE/ACM Transactions on Networking, vol. 18, no. 5, pp. $1530-1543,2010$

[10] Z. Li, L. Sun, and E. Ifeachor, "Adaptive multi-copy routing for intermittently connected mobile ad hoc networks," in IEEE GLOBECOM '06, San Francisco, California, USA, 2006.

[11] A. Jindal and K. Psounis, "Optimizing multi-copy routing schemes for resource constrained intermittently connected mobile networks," in IEEE ACSSC '06, Singapore, 2006.

[12] A. Lindgren, A. Doria, and O. Schelén, "Probabilistic routing in intermittently connected networks," ACM SIGMOBILE Mob. Comput. Commun. Rev., vol. 7, pp. 19-20, 2003.

[13] http://www.netlab.tkk.fi/tutkimus/dtn/theone/. 